\title{
Change in Pseudomonas aeruginosa prevalence in cystic fibrosis adults over time
}

\author{
Mathew R. Crull ${ }^{1,4^{*}}$, Kathleen J. Ramos ${ }^{1}$, Ellen Caldwell ${ }^{1}$, Nicole Mayer-Hamblett ${ }^{2,3}$, Moira L. Aitken ${ }^{1}$ \\ and Christopher H. Goss ${ }^{1,2}$
}

\begin{abstract}
Background: Little is known about risk factors for chronic and mucoid Pseudomonas aeruginosa $(\mathrm{Pa})$ infection in cystic fibrosis (CF) adults, and whether the prevalence is changing.

Methods: We employed a retrospective cohort to analyze data from a single adult CF center (2002 to 2012). Regression models were used to assess independent predictors and change in prevalence of chronic and mucoid Pa infection over time.

Results: The odds ratio of mucoid Pa infection was significantly less in individuals with better baseline lung function (OR 0.84,95\%Cl:0.77-0.92) and those diagnosed after the age of 25 (OR 0.21,95\%Cl:0.05-0.95). The prevalence of chronic Pa and mucoid Pa decreased during the time interval. After adjusting for confounders, the observed decrease in chronic and mucoid Pa between 2002 and 2012 was no longer significant.
\end{abstract}

Conclusions: The prevalence of chronic and mucoid Pa is decreasing. Larger studies are needed to confirm these regional trends and their significance.

Keywords: Cystic fibrosis, Pseudomonas aeruginosa, Chronic infection, Epidemiology, Temporal trends

\section{Background}

Cystic Fibrosis (CF) is the most common severe monogenic disorder among Caucasians [1]. CF was once considered a fatal childhood disease, but with advances in care, survival has steadily improved. Mortality decreased by $1.8 \%$ per year between the years 2000 to 2012, and if this observed rate continues, individuals born with CF in 2010 are predicted to have a median life expectancy of more than 50 years [2]. In 2013, half of the US CF population was 18 years of age or older [3]. Chronic lung disease in CF, however, continues to cause significant morbidity, and respiratory failure is the leading cause of death in this population [4]. Chronic endobronchial infections are central in the pathogenesis of chronic lung disease [5].

\footnotetext{
* Correspondence: mrcrull@uw.edu

${ }^{1}$ Department of Medicine, University of Washington, Seattle, WA, USA

${ }^{4}$ University of Washington Medical Center, Campus Box 3565221959 N.E.

Pacific, Seattle, WA 98195, USA

Full list of author information is available at the end of the article
}

Pseudomonas aeruginosa $(\mathrm{Pa})$ is the most common cultured respiratory pathogen in $\mathrm{CF}$, and in children, $\mathrm{Pa}$ is associated with a more rapid decline in lung function and worse survival [6-8]. Prior reports estimated that by age eighteen, $80 \%$ of individuals with CF are infected with $\mathrm{Pa}$ [9]. Over time, most individuals with CF become chronically infected with $\mathrm{Pa}$, and a mucoid phenotype of $\mathrm{Pa}$ frequently becomes the predominant form found in culture $[10,11]$. Chronic and mucoid Pa infections are associated with increased morbidity and mortality when compared to individuals not infected with $\mathrm{Pa}[12,13]$. Additionally, due to formation of biofilms, development of antibiotic resistance and overall bacterial abundance, this form of $\mathrm{Pa}$ infection is challenging to eradicate $[5,14,15]$.

Because of the challenges with eradicating chronic and mucoid $\mathrm{Pa}$, the focus of treatment has been on preventing the chronic infection [16]. Recent studies in children demonstrated eradication of newly acquired Pa from airway is associated with delayed reacquisition of $\mathrm{Pa}$ even after stopping antibiotics [17-20]. These studies suggest 
that development of chronic Pa infection might be decreasing, but this has never been formally investigated in the adult CF population. Using the CF Foundation Patient Registry a recent study showed a decline in both the incidence and prevalence of $\mathrm{Pa}$ between 2006 and 2012 [21]. This study, however, did not look specifically at chronic or mucoid Pa infection.

We hypothesize the prevalence of chronic and mucoid Pa lung infection in the older CF population is decreasing with time and there are independent predictors of chronic and mucoid $\mathrm{Pa}$ infection in $\mathrm{CF}$ adults, which may be unique compared to the younger CF population. Using a longitudinal adult $\mathrm{CF}$ cohort, we sought to assess the change in both chronic and mucoid Pa infections between 2002 and 2012 and identify independent predictors of the chronically infected and mucoid Pa states.

\section{Methods}

Population

A retrospective cohort study design was employed using individuals age 18 or older receiving care through the Adult CF Clinic at the University of Washington Medical Center between 2002 and 2012. Patients were able to contribute data from multiple years; however, all individuals with no evaluable culture data during a 1-year period were excluded for the calendar year in which the data was absent. No imputation of missing data was performed. Sensitivity analyses were performed to assess the impact of missing data.

\section{Definitions of predictors of interest}

Both time independent and time dependent variables were considered as potential independent predictors of chronic and mucoid Pa. Time independent variables included age at diagnosis, gender, CF mutation status, CF transmembrane conductance regulator (CFTR) function and baseline lung function. Time dependent covariates included age, CF-related diabetes (CFRD) and pancreatic insufficiency (PI). Age at diagnosis was examined both as a continuous and binary variable. As a binary variable, age at diagnosis was categorized as 25 years of age or younger and greater than 25 years of age. CFTR function was classified as minimal (class I, II, and III), and residual (class IV and V). All remaining genotypes were grouped together along with those with unknown mutations. The residual CFTR function group was used as the comparator group. CFRD was defined by receiving insulin, and PI was defined as receiving pancreatic enzymes. Percent predicted forced expiratory volume in one second $\left(\mathrm{FEV}_{1}\right)$ at time of cohort entry was used to define baseline lung function. When analyzing the change in prevalence of both chronic $\mathrm{Pa}$ and mucoid $\mathrm{Pa}$, potential confounding variables included age, age of diagnosis, gender, CFTR function, and baseline lung function. The inclusion of confounding variables was made a priori.

Anticipating relatively small numbers of individuals within each year, calendar time was divided into four time periods (2002-2004, 2005-2007, 2008-2010, and 2011-2012) for analysis of change in prevalence. This decision was made prior to analysis of the data. This division of the observation period balanced the number of subjects within each time period.

\section{Outcome definitions}

The primary outcomes of interest for identification of risk factors and change in prevalence were chronic $\mathrm{Pa}$ and mucoid $\mathrm{Pa}$ infection. Due to the frequency of respiratory culturing in this cohort, chronic Pa infection was defined using a modified definition of chronic Pa initially proposed by Lee et al. [22]. Individuals were defined as chronic when three or more respiratory cultures were provided in a calendar year and $50 \%$ or more were positive for Pa. If only two cultures were obtained in a calendar year, both cultures needed to be positive for Pa to be considered chronically infected. Those individuals who provided less than 2 cultures within a calendar year were excluded from the primary analysis. A sensitivity analysis was performed including all subjects regardless of the number of cultures provided within a year. Mucoid $\mathrm{Pa}$ was defined having at least one respiratory culture with the mucoid phenotype during a calendar year. Sensitivity analyses looking at the influence of CFTR function, duration of time periods, changes in cohort characteristics and inhaled antibiotic use were also performed.

\section{Analysis}

Descriptive statistics were used to describe the cohort. Continuous variables were reported as medians with inter quartile ranges and categorical variables were reported as proportions. To account for repeated measurements, population averaged generalized estimating equation (GEE) regression models with independent working correlation were used to assess independent predictors and change in prevalence of both chronic and mucoid $\mathrm{Pa}$ infection over time [23]. When assessing independent predictors of chronic and mucoid $\mathrm{Pa}$ infection, a univariate regression model was first used to screen for significant variables. Those variables with a significance level less than or equal to 0.1 were then included in a multivariable regression model that included an indicator for time period to account for changes in cohort over the observation period. Both univariate and multivariable regression analysis were used when assessing the change in prevalence of chronic and mucoid Pa over time. Odds ratio with a $95 \%$ confidence interval was used to report results of the GEE models. Unless otherwise noted, a two-tailed P-value, not adjusted for multiple comparisons, less than 0.05 was 
considered statistically significant. All analyses were performed using STATA version 13 (StataCorp LP, College Station, Texas). The University of Washington Institutional Review Board approved this study (45798).

\section{Results}

\section{Overview of cohort}

During the observation period, data was collected from 402 individuals. The average observation period was 4.6 years (range $1-11$ years). Demographic data for the cohort from the year of entry is summarized in Table 1 . This cohort was dynamic with individuals aging or migrating into the population throughout the observation period. Additionally, some individuals left the cohort due to death or out-migration. Changes in population characteristics during the observation period are summarized in Additional file 1: Table E1. The cohort increased from 123 patients in 2002 to 211 patients in 2012 .

Table 1 Characteristics of cohort at time of entry into the cohort

\begin{tabular}{|c|c|}
\hline & $(N=402)$ \\
\hline Mean age, years (SD) & $29.7(9.4)$ \\
\hline Median age of diagnosis, years (IQR) & $1.5(8)$ \\
\hline Gender, \% Female (N) & $48.8(196)$ \\
\hline Race, \% Caucasian (N) & $94.3(379)$ \\
\hline Ethnicity, \% Hispanic (N) & $1.9(7)$ \\
\hline \multicolumn{2}{|l|}{ f508del status } \\
\hline Homozygous, \% (N) & $52.4(200)$ \\
\hline Heterozygous, \% (N) & $39.0(149)$ \\
\hline Other, \% (N) & $8.6(33)$ \\
\hline \multicolumn{2}{|l|}{ CFTR Mutation Classification ${ }^{\text {ad }}$} \\
\hline Minimal, \% (N) & $71.7(274)$ \\
\hline Residual, \% (N) & $10.7(41)$ \\
\hline Unclassified, \% (N) & $17.5(67)$ \\
\hline $\mathrm{FEV}_{1}, \%$ predicted $(\mathrm{SD})^{\mathrm{d}}$ & $63.5(26.0)$ \\
\hline \multicolumn{2}{|l|}{ Co-morbidities } \\
\hline CF-related diabetes ${ }^{b d}, \%(N)$ & $28.0(109)$ \\
\hline Pancreatic insufficiency ${ }^{c d}, \%(\mathrm{~N})$ & 86.3 (308) \\
\hline Inhaled Antibiotic Use ${ }^{d}$ & $49.5(147)$ \\
\hline \multicolumn{2}{|l|}{ Microbiology } \\
\hline Any form of Pseudomonas isolated, \% (N) & $64.2(238)$ \\
\hline Mucoid Pseudomonas phenotype, \% (N) & $48.0(178)$ \\
\hline \multicolumn{2}{|c|}{$\begin{array}{l}\text { SD standard deviation, IQR inter quartile range } \\
\text { a Minimal: Both alleles containing mutations resulting in minimal CFTR function } \\
\text { (Class 1, 2, or 3); Residual: at least one allele containing mutation resulting in } \\
\text { partial CFTR function (Class } 4 \text { or 5); Unclassified: at least one allele with } \\
\text { unknown CFTR function and if other allele function known, mutation resulting } \\
\text { in minimal CFTR function } \\
{ }^{6} \text { CF-related diabetes: use of insulin } \\
\text { CPancreatic insufficiency: use of pancreatic enzymes } \\
\text { dMissing data (N, \%): CFTR Mutation Classification ( } 20,5.0 \%), F E V_{1}(47,11.7 \%) \text {, } \\
\text { CFRD (12,3.0\%), PI (45, } 11.2 \%) \text {, Inhaled antibiotic use }(105,26 \%)\end{array}$} \\
\hline
\end{tabular}

Genotype data was available on 382 individuals (95\%) in the cohort. Over the 11 years, the proportion of individuals homozygous for f508del decreased from 57.7 to $48.8 \%$. Mean population percent-predicted $\mathrm{FEV}_{1}$ increased from 59.0 to $68.0 \%$ of predicted. The proportion using an inhaled anti-pseudomonal antibiotic remained relatively constant during the observation period. A cross sectional comparison of cohort demographics between 2002 and 2012 based on state of Pa infection and number of cultures is presented in Additional file 1: Table E2 and E3 respectively.

\section{Risk factors for chronic and mucoid Pa}

Results of the univariate and multivariable analysis for independent predictors of chronic Pa infection using a modified Leeds definition and mucoid $\mathrm{Pa}$ are in Table 2. Subjects with worse baseline lung function were significantly more likely to have mucoid $\mathrm{Pa}(P$-value $<0.001)$. When comparing two groups with a $10 \%$ difference in baseline lung function, the odds of having a mucoid $\mathrm{Pa}$ infection was $16 \%$ less in the group with better lung function (OR: 0.84, 95\% CI: 0.77-0.92). Those individuals diagnosed after the age of 25 were less likely to have a mucoid Pa infection (OR: 0.21, 95\% CI: 0.05-0.95). All

Table $\mathbf{2}$ Independent predictors of chronic and mucoid Pa infection

\begin{tabular}{|c|c|c|}
\hline & Chronic $\mathrm{Pa}$ & Mucoid Pa \\
\hline & OR $(95 \% \mathrm{Cl})$ & OR $(95 \% \mathrm{Cl})$ \\
\hline \multicolumn{3}{|l|}{ Univariate } \\
\hline Age (yr) & $0.83(0.63-1.10)$ & $0.82(0.66-1.02)$ \\
\hline \multicolumn{3}{|l|}{ Diagnosis } \\
\hline Age (yr) & $0.96(0.94-0.98)$ & $0.97(0.96-0.99)$ \\
\hline Diagnosis after 25 years of age & $0.12(0.04-0.31)$ & $0.21(0.09-0.46)$ \\
\hline Gender, female & $1.07(0.65-1.77)$ & $0.94(0.61-1.44)$ \\
\hline CFTR function ${ }^{a}$ & $1.94(1.10-3.44)$ & $2.61(1.28-5.63)$ \\
\hline CF-related diabetes ${ }^{\mathrm{b}}$ & $1.02(0.63-1.65)$ & $0.88(0.57-1.34)$ \\
\hline Pancreatic insufficiency ${ }^{c}$ & $2.21(1.11-4.37)$ & $2.52(1.46-4.38)$ \\
\hline Lung Function $^{d}$ & $0.90(0.82-1.00)$ & $0.79(0.71-0.88)$ \\
\hline \multicolumn{3}{|l|}{ Multivariable $\mathrm{e}^{\mathrm{e}}$} \\
\hline Age (yr) & $1.03(0.99-1.08)$ & $1.00(0.97-1.03)$ \\
\hline \multicolumn{3}{|l|}{ Diagnosis } \\
\hline Age (yr) & $0.96(0.91-1.02)$ & $1.01(0.97-1.05)$ \\
\hline Diagnosis after 25 years of age & $0.18(0.03-1.20)$ & $0.21(0.05-0.95)$ \\
\hline CFTR function ${ }^{\mathrm{a}}$ & $0.51(0.17-1.55)$ & $0.99(0.42-2.34)$ \\
\hline Pancreatic insufficiency ${ }^{c}$ & $1.23(0.53-2.82)$ & $1.58(0.89-2.80)$ \\
\hline Lung Function $^{d}$ & $0.91(0.82-1.01)$ & $0.84(0.77-0.92)$ \\
\hline
\end{tabular}

${ }^{a}$ Comparator group: Residual CFTR function: at least one allele containing mutation resulting in partial CFTR function (Class 4 or 5)

${ }^{\mathrm{b}} \mathrm{CF}$-related diabetes: use of insulin

'Pancreatic insufficiency: use of pancreatic enzymes

$\mathrm{d}_{10 \%}$ difference in percent predicted $\mathrm{FEV}_{1}$

${ }^{e}$ Variables with significance level $\leq 0.1$ included in multivariable analysis 
other variables included in both the mucoid $\mathrm{Pa}$ and chronic Pa multivariable models did not reach a significance level less than 0.05 (Table 2).

\section{Prevalence of chronic Pa over time}

The prevalence of chronic $\mathrm{Pa}$ in those who provided at least two respiratory cultures in a calendar year decreased from $73.5 \%$ in 2002 to $67.0 \%$ in 2012 (Additional file 1: Table E1). The average prevalence of chronic Pa infection decreased by $5.9 \%$ from the 2002 to 2004 time period to the 2011 to 2012 time period (Fig. 1). After adjusting for gender, age, age at diagnosis, CFTR function and baseline lung function, the odds ratio of chronic Pa infection in the 2011-2012 time period relative to 2002-2004 time period was 0.82 (95\% CI: 0.31-2.14) (Table 3). Relative to the 2002-2004 time period, odds ratio of chronic Pa infection was not significantly different than other time periods. In both the unadjusted and adjusted models of chronic Pseudomonas infection, the P-values of the trend test for time period were not significant (Test of trend: unadjusted $P=0.25$; adjusted $P=0.84$ ) (Table 3 ).

\section{Prevalence of mucoid Pa over time}

The prevalence of isolating the mucoid phenotype of $\mathrm{Pa}$ on respiratory culture in 2002 was $68.2 \%$ and in 2012 the prevalence was 58.1\% (Additional file 1: Table E1). The mean prevalence of mucoid Pa decreased by $10.3 \%$ when comparing the 2002-2004 time period and the 2011-2012 time period (Fig. 1). When comparing the 2011-2012 time period to the 2002-2004 time period, the odds ratio of isolating the mucoid Pa phenotype in culture was 0.64 (95\% CI: $0.43-0.96)$ with a significant test of trend $(p=0.04)$, but after adjusting for potential confounding the change was no longer significant (OR 0.55; 95\% CI: 0.27-1.11) (Table 4).
Table 3 Odds ratio for prevalence of chronic Pseudomonas aeruginosa infection using modified Leeds criteria

\begin{tabular}{lll}
\hline Year & $\operatorname{OR}^{\mathrm{a}}(95 \% \mathrm{Cl})$ & $\begin{array}{l}\text { Adjusted } \\
\mathrm{OR}^{\mathrm{b}, \mathrm{c}}(95 \% \mathrm{Cl})\end{array}$ \\
\hline $2002-2004$ & - & - \\
$2005-2007$ & $1.00(0.65-1.53)$ & $1.22(0.42-3.52)$ \\
$2008-2010$ & $0.97(0.60-1.56)$ & $1.29(0.55-3.00)$ \\
$2011-2012$ & $0.75(0.46-1.21)$ & $0.82(0.31-2.14)$ \\
\hline
\end{tabular}

a Trend test p: 0.25

${ }^{\mathrm{b}}$ Trend test p: 0.85

${ }^{\mathrm{C}}$ Adjusted for age, gender, age at diagnosis, CFTR function (minimal: class 1, 2, 3; residual: class 4, 5; Unclassified), baseline lung function (percent predicted FEV1)

Demographic data was similar between the chronic Pa by modified Leeds definition and the mucoid Pa phenotype group in 2002 and 2012 (Additional file 1: Table E4).

\section{Sensitivity analysis}

When restricting the cohort to only individuals homozygous for the f508del mutation, a trend toward decreasing prevalence of both chronic and mucoid Pa was observed (Additional file 1: Table E5). This trend was not significant for either group. Inclusion of all individuals who provided less two respiratory cultures within a year as free of chronic infection resulted in a significant decrease in the prevalence of chronic Pa infection (Test of trend P-value 0.002) (Additional file 1: Table E6). Adjusting for inhaled antibiotic use did not significantly change the observed trend in prevalence of chronic or mucoid Pa infection (Additional file 1: Table E7). Using a two time period model (2002-2007, 2008-2012), the odds of infection was decreased but not significantly in the later time period relative to the early period for both chronic

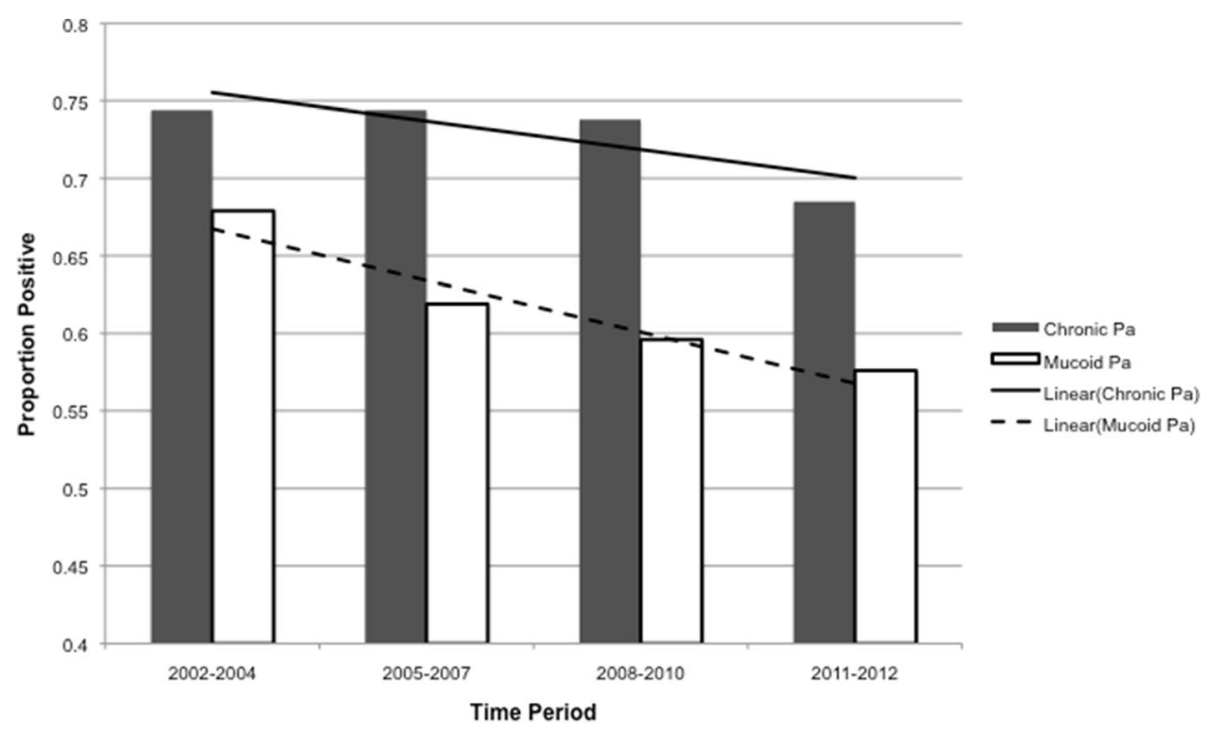

Fig. 1 By time period, prevalence of chronic Pa by modified Leeds Criteria and mucoid Pa 
Table 4 Odds ratio for prevalence of mucoid Pseudomonas aeruginosa phenotype in culture

\begin{tabular}{lll}
\hline Year & $\mathrm{OR}^{\mathrm{a}}(95 \% \mathrm{Cl})$ & $\begin{array}{l}\text { Adjusted } \\
\mathrm{OR}^{\mathrm{b}, \mathrm{c}}(95 \% \mathrm{Cl})\end{array}$ \\
\hline $2002-2004$ & - & - \\
$2005-2007$ & $0.77(0.56-1.06)$ & $0.81(0.40-1.67)$ \\
$2008-2010$ & $0.70(0.57-1.03)$ & $0.77(0.39-1.52)$ \\
$2011-2012$ & $0.64(0.43-0.96)$ & $0.55(0.27-1.11)$ \\
\hline
\end{tabular}

a Trend test p: 0.04

${ }^{\mathrm{b}}$ Trend test p: 0.11

${ }^{c}$ Adjusted for age, gender, age at diagnosis, CFTR function (minimal: class 1, 2, 3; residual: class 4, 5; Unclassified), baseline lung function (percent predicted $\mathrm{FEV}_{1}$ )

(OR 0.86; 95\% CI: 0.62-1.21) and mucoid (0.78; 95\% CI: 0.59-1.02) Pa infections.

\section{Discussion}

Respiratory failure is the leading cause of death in CF, and $\mathrm{Pa}$ infection, particularly chronic and the mucoid phenotype, has been associated with increased morbidity and mortality $[7,10,12,13]$. Using a single center adult CF cohort, we observed a decrease in prevalence of both chronic and mucoid $\mathrm{Pa}$ over an 11-year observation period. In a multivariable model adjusting for potential confounding and repeated usage of patients, the change in prevalence of both chronic and mucoid $\mathrm{Pa}$, however, did not meet the pre-specified significance level of 0.05 . We found age of diagnosis, CFTR function, PI and lung function to be predictors of both chronic and mucoid Pa when using a univariate analysis. Age of diagnosis and baseline lung function remained significant independent predictors of mucoid $\mathrm{Pa}$ infection after adjusting our analysis for potential confounding. This suggests that in the adult CF population individuals are less likely to have a mucoid $\mathrm{Pa}$ infection when they are either diagnosed at a later age or have preserved lung function.

While our observed change in Pa over time may be due to chance, a possible explanation for the decreasing prevalence over time is a change in the population characteristics of this cohort. The hypothesis is supported when looking at the change in mucoid Pa prevalence during the observation period. A significant change in prevalence over time was observed in the unadjusted analysis of mucoid Pa. This association was no longer significant after adjusting for clinical and demographic variables that may confound the association between time and change in prevalence. Comparing all individuals making up the cohort in 2002 to 2012, the proportion of individuals in the cohort homozygous for f508del decreased. Along with this change there was an increase in the proportion of individuals with an unknown CFTR functional class. With genetic testing, the proportion of adults diagnosed with $\mathrm{CF}$ has increased, and of these newly diagnosed adults, fewer are homozygous for f508del [24]. Residual CFTR function may result in less chronic infection. A recent study demonstrated a decrease in the prevalence of $\mathrm{Pa}$ in respiratory cultures in a group of individuals with the G551D mutation receiving ivacaftor. CFTR having a role in innate host response to infection was a postulated explanation [25]. While CFTR function was not associated with chronic or mucoid $\mathrm{Pa}$ infections in our single center cohort, the degree of CFTR function may play an important role in development of chronic and mucoid $\mathrm{Pa}$ infections and warrants further exploration. Lastly, individuals with worse lung function were more likely to have chronic forms of $\mathrm{Pa}$ infection. The observed improvement in lung function during the observation period may also explain the trend in prevalence of both mucoid and chronic Pa.

Restricting the cohort to individuals homozygous for the f508del mutation resulted in a trend, albeit nonsignificant, toward lower prevalence of both chronic and mucoid $\mathrm{Pa}$. This trend suggests influences aside from change in cohort demographics might be contributing to the change in chronic Pa prevalence. One such influence is the implementation of inhaled anti-pseudomonal antibiotics. Multiple prospective studies involving the use of inhaled and oral anti-pseudomonal antibiotics have demonstrated clearance of newly acquired Pa from respiratory culture $[17,18,26]$. Eradication of this form of $\mathrm{Pa}$ from the airways has been associated with improved lung function, fewer pulmonary exacerbations and improved survival $[19,27]$. Eradicating newly acquired $\mathrm{Pa}$ with inhaled antibiotics is now the standard of care [28]. In the pediatric population, the practice of inhaled antipseudomonal antibiotics to eradicate new acquisition of $\mathrm{Pa}$ has been associated with a decline in prevalence of chronic Pa infection [29-31]. Whether the attempts to eradicate newly acquired $\mathrm{Pa}$ from the airways has translated into a reduction in the frequency of mucoid and chronic Pa in adults with CF is unknown. Our study is the first to analyze change in prevalence of mucoid and chronic Pa in $\mathrm{CF}$ adults.

Knowledge about the factors associated with chronic Pa can improve care in $\mathrm{CF}$ adults. Identifying risk factors for development of chronic Pa can tailor care and ultimately improve quality of life. In a prospective cohort study, CFTR genotype, female gender, age of diagnosis, and pancreatic enzyme use were identified as risk factors for initial $\mathrm{Pa}$ acquisition in children [32]. Predictors of chronic $\mathrm{Pa}$, however, have not been well described in the literature. The limited literature may in part be due to no universally agreed upon definition for chronic $\mathrm{Pa}$. A variable amount of time may lapse between first acquisition of $\mathrm{Pa}$ and chronic $\mathrm{Pa}$ status, and aggressive attempts to eradicate newly acquired $\mathrm{Pa}$ from the airways have lengthened the 
time between new acquisition and chronic $\mathrm{Pa}$ [30]. This may limit our ability to extrapolate risk factors from newly acquired $\mathrm{Pa}$ to chronic $\mathrm{Pa}$. The mucoid phenotype of $\mathrm{Pa}$ has been associated with increased morbidity and mortality in CF [12,13] and has been associated with onset of chronic Pa [33]. In children, gender, lower baseline lung function, genotype and absence of MRSA in sputum culture are risk factors associated with acquisition of the mucoid $\mathrm{Pa}$ in respiratory culture [34]. Our study is the first to describe independent predictors of chronic and mucoid $\mathrm{Pa}$ infection in the adult CF population in the post-pediatric inhaled tobramycin era. Of the predictors, baseline lung function and being diagnosed with $\mathrm{CF}$ as an adult appear to be the predominant predictors not being infected with mucoid or chronic Pa. Being diagnosed with $\mathrm{CF}$ as an adult is likely reflective of having a milder phenotype, which may correlate with degree of functional CFTR [24]. Whether the predictors found in our study translate to risk factors for the development of chronic and mucoid Pa still needs to be determined.

There are several limitations to consider with this study. Patients cared for at a single CF center were used to conduct this analysis. This both limits generalizability and the ability to detect smaller but clinically meaningful changes in prevalence. Due to challenges eradicating chronic Pa from the airways, a longer observation period may be needed to detect a significant change in the prevalence. To increase the number of observations within each time period, a sensitivity analysis was performed using only two time periods (2002-2007 and 2008-2012). This analysis showed a similar trend toward decreasing prevalence but statistical significance was not met. Incidence of developing chronic Pa may be a better measurement of change, as it would not be influenced by the challenges of eradicating chronic $\mathrm{Pa}$. Our definition of chronic Pa used a modified form of the Leeds criteria and relied on the number of cultures obtained within a calendar year. Under the Leeds definition, chronic $\mathrm{Pa}$ was defined as having respiratory cultures positive for $\mathrm{Pa}$ in more than $50 \%$ of the months within a 12 month period. Collecting respiratory cultures at least every 3 months was recommended when applying this definition [22]. In our adult cohort, the frequency of respiratory cultures on average was less than quarterly. Application of the Leeds definition could have resulted in significant misclassification due too few cultures collected during the year. To reduce misclassification, the analysis restricted the cohort to only include individuals with two or more cultures in a calendar year. This restriction reduced the number of observations available for analysis, which further limited the study's ability to detect a significant change in prevalence. In our sensitivity analysis using all subjects irrespective of annual culture numbers, a significant decrease in the prevalence of chronic Pa was observed even after adjusting for other variables. This observation suggests that in a larger cohort the trend observed may be significant.

\section{Conclusion}

This analysis is one of the first in recent times to identify independent predictors of mucoid and chronic Pa infection in CF adults and analyze how the prevalence of both mucoid and chronic Pa infection in CF adults have changed with time. What appears to be a decrease in the frequency of chronic and mucoid $\mathrm{Pa}$ in recent years warrants further study at a national level to confirm these regional trends and their significance.

\section{Additional file}

Additional file 1: VELVET: list of study investigators and central ethics committees. (DOCX $21 \mathrm{~kb})$

\section{Abbreviations}

CF: Cystic Fibrosis; CFRD: Cystic fibrosis related diabetes; CFTR: Cystic fibrosis transmembrane conductance regulator; $\mathrm{FEV}_{1}$ : Forced expiratory volume in one second; GEE: Generalized estimating equation; Pa: Pseudomonas aeruginosa; PI: Pancreatic insufficiency

\section{Acknowledgement \\ The authors thank the Cystic Fibrosis Foundation and Dr Bruce Marshall for providing clinical data.}

\section{Funding}

$\mathrm{MC}$ receives funding from the Cystic Fibrosis Foundation (Second Year Clinical Fellowship Award) CG receives funding from the Cystic Fibrosis Foundation, the NIH (R01HL103965, R01 Al101307, P30 DK089507) and the FDA (R01 FD003704).

\section{Availability of data and materials}

The dataset generated and analyzed during the current study are not publicly available. The Cystic Fibrosis Foundation Patient Registry is not a public data set. Investigators can apply to use the data by completing a data request application from the Cystic Fibrosis Foundation. One requirement of a data request application is that the investigator on the research team must be associated with a US Cystic Fibrosis Foundation accredited Cystic Fibrosis Care Center.

\section{Authors' contributions}

MC, NMH and CG made substantial contributions to conception and design, or acquisition of data, or analysis and interpretation of data. All authors were involved in drafting the manuscript or revising it critically for important intellectual content. All authors read and approved the final manuscript.

\section{Competing interests}

The authors declare that they have no competing interests.

\section{Consent for publication}

Not applicable.

Ethics approval and consent to participate

The University of Washington Institutional Review Board approved this study (45798).

\section{Author details}

'Department of Medicine, University of Washington, Seattle, WA, USA. ${ }^{2}$ Department of Pediatrics, Division of Pulmonary, University of Washington, Seattle, WA, USA. ${ }^{3}$ Department of Biostatistics, University of Washington, Seattle, WA, USA. ${ }^{4}$ University of Washington Medical Center, Campus Box 3565221959 N.E. Pacific, Seattle, WA 98195, USA. 
Received: 5 July 2016 Accepted: 22 November 2016 Published online: 07 December 2016

\section{References}

1. Rogers GB, Hart CA, Mason JR, Hughes M, Walshaw MJ, Bruce KD. Bacterial Diversity in Cases of Lung Infection in Cystic Fibrosis Patients: 16S Ribosomal DNA (rDNA) Length Heterogeneity PCR and 165 rDNA Terminal Restriction Fragment Length Polymorphism Profiling. J Clin Microbiol. 2003;41(8):3548-58.

2. MacKenzie T, Gifford AH, Sabadosa KA, Quinton HB, Knapp EA, Goss CH, et al. Longevity of Patients With Cystic Fibrosis in 2000 to 2010 and Beyond: Survival Analysis of the Cystic Fibrosis Foundation Patient Registry. Ann Intern Med. 2014;161(4):233-41.

3. Cystic Fibrosis Foundation Patient Registry: 2013 Annual Data Report. Bethesda, MD: Cystic Fibrosis Foundation; 2014.

4. Ratjen F, Döring G. Cystic fibrosis. Lancet. 2003;361(9358):681-9.

5. Treggiari MM, Rosenfeld M, Retsch-Bogart G, Gibson R, Ramsey B. Approach to eradication of initial Pseudomonas aeruginosa infection in children with cystic fibrosis. Pediatr Pulmonol. 2007;42(9):751-6.

6. Konstan MW, Morgan WJ, Butler SM, Pasta DJ, Craib ML, Silva SJ, et al. Risk factors for rate of decline in forced expiratory volume in one second in children and adolescents with cystic fibrosis. J Pediatr. 2007;151(2):134-9. 139.e1.

7. Emerson J, Rosenfeld M, McNamara S, Ramsey B, Gibson RL. Pseudomonas aeruginosa and other predictors of mortality and morbidity in young children with cystic fibrosis. Pediatr Pulmonol. 2002;34(2):91-100.

8. Nixon GM, Armstrong DS, Carzino R, Carlin JB, Olinsky A, Robertson CF, et al. Clinical outcome after early Pseudomonas aeruginosa infection in cystic fibrosis. J Pediatr. 2001;138(5):699-704.

9. Cystic Fibrosis Foundation Patient Registry: 2012 Annual Data Report. Bethesda, MD: Cystic Fibrosis Foundation; 2013.

10. Li Z, Kosorok M, Farrell PM, et al. Longitudinal development of mucoid pseudomonas aeruginosa infection and lung disease progression in children with cystic fibrosis. JAMA. 2005;293(5):581-8.

11. LiPuma JJ. The Changing Microbial Epidemiology in Cystic Fibrosis. Clin Microbiol Rev. 2010;23(2):299-323

12. Henry RL, Mellis CM, Petrovic L. Mucoid Pseudomonas aeruginosa is a marker of poor survival in cystic fibrosis. Pediatr Pulmonol. 1992;12(3):158-61.

13. Parad RB, Gerard CJ, Zurakowski D, Nichols DP, Pier GB. Pulmonary Outcome in Cystic Fibrosis Is Influenced Primarily by Mucoid Pseudomonas aeruginosa Infection and Immune Status and Only Modestly by Genotype. Infect Immun. 1999;67(9):4744-50.

14. Costerton JW, Stewart PS, Greenberg EP. Bacterial biofilms: a common cause of persistent infections. Science. 1999;284(5418):1318-22.

15. Staudinger BJ, Muller JF, Halldórsson S, Boles B, Angermeyer A, Nguyen D, et al. Conditions Associated with the Cystic Fibrosis Defect Promote Chronic Pseudomonas aeruginosa Infection. Am J Respir Crit Care Med. 2014;189(7):812-24.

16. Mogayzel PJ, Naureckas ET, Robinson KA, Mueller G, Hadjiliadis D, Hoag JB, et al. Cystic Fibrosis Pulmonary Guidelines. Am J Respir Crit Care Med. 2013; 187(7):680-9.

17. Ratjen F, Munck A, Kho P, Angyalosi G. Treatment of early Pseudomonas aeruginosa infection in patients with cystic fibrosis: the ELITE trial. Thorax. 2010;65(4):286-91.

18. Treggiari MM, Retsch-Bogart G, Mayer-Hamblett N, Khan U, Kulich M, Kronmal $\mathrm{R}$, et al. Comparative efficacy and safety of 4 randomized regimens to treat early Pseudomonas aeruginosa infection in children with cystic fibrosis. Arch Pediatr Adolesc Med. 2011;165(9):847.

19. Mayer-Hamblett N, Kronmal RA, Gibson RL, Rosenfeld M, Retsch-Bogart G, Treggiari MM, et al. Initial Pseudomonas aeruginosa treatment failure is associated with exacerbations in cystic fibrosis. Pediatr Pulmonol. 2012;47(2): $125-34$

20. Zemanick ET, Emerson J, Thompson V, McNamara S, Morgan W, Gibson RL, et al. Clinical outcomes after initial pseudomonas acquisition in cystic fibrosis. Pediatr Pulmonol. 2014;50(1):42-8.

21. Knapp EA, Salsgiver E, Fink A, Sewall A, Marshall BC, Saiman L. Trends in respiratory microbiology of people with cystic fibrosis in the united states, 2006-2012. Pediatr Pulmonol. 2014;49(S38):317.

22. Lee TWR, Brownlee KG, Conway SP, Denton M, Littlewood JM. Evaluation of a new definition for chronic Pseudomonas aeruginosa infection in cystic fibrosis patients. J Cyst Fibros Off J Eur Cyst Fibros Soc. 2003;2(1):29-34.

23. Hanley JA, Negassa A, Edwardes MD deB, Forrester JE. Statistical Analysis of Correlated Data Using Generalized Estimating Equations: An Orientation. Am J Epidemiol. 2003; 157(4):364-375.
24. Keating $C L$, Liu X, DiMango EA. Classic respiratory disease but atypical diagnostic testing distinguishes adult presentation of cystic fibrosis. CHEST J. 2010;137(5):1157-63.

25. Rowe SM, Heltshe SL, Gonska T, Donaldson SH, Borowitz D, Gelfond D, et al. Clinical Mechanism of the Cystic Fibrosis Transmembrane Conductance Regulator Potentiator Ivacaftor in G551D-mediated Cystic Fibrosis. Am J Respir Crit Care Med. 2014;190(2):175-84.

26. Gibson RL, Emerson J, McNamara S, Burns JL, Rosenfeld M, Yunker A, et al. Significant microbiological effect of inhaled tobramycin in young children with cystic fibrosis. Am J Respir Crit Care Med. 2003;167(6):841-9.

27. Frederiksen B, Koch C, Høiby N. Antibiotic treatment of initial colonization with Pseudomonas aeruginosa postpones chronic infection and prevents deterioration of pulmonary function in cystic fibrosis. Pediatr Pulmonol. 1997;23(5):330-5.

28. Mogayzel PJ, Naureckas ET, Robinson KA, Brady C, Guill M, Lahiri T, et al. Cystic Fibrosis Foundation Pulmonary Guideline. Pharmacologic Approaches to Prevention and Eradication of Initial Pseudomonas aeruginosa Infection. Ann Am Thorac Soc. 2014;11(10):1640-50.

29. Lee TWR, Brownlee KG, Denton M, Littlewood JM, Conway SP. Reduction in prevalence of chronic Pseudomonas aeruginosa infection at a regional pediatric cystic fibrosis center. Pediatr Pulmonol. 2004;37(2):104-10.

30. Hansen CR, Pressler T, Høiby N. Early aggressive eradication therapy for intermittent Pseudomonas aeruginosa airway colonization in cystic fibrosis patients: 15 years experience. J Cyst Fibros. 2008;7(6):523-30.

31. Frederiksen B, Koch C, Hoeiby N. Changing epidemiology of Pseudomonas aeruginosa infection in Danish cystic fibrosis patients (1974-1995). Pediatr Pulmonol. 1999;28(3):159-66.

32. Rosenfeld M, Emerson J, McNamara S, Thompson V, Ramsey BW, Morgan W, et al. Risk factors for age at initial Pseudomonas acquisition in the cystic fibrosis epic observational cohort. J Cyst Fibros. 2012;11(5):446-53.

33. Pressler T, Frederiksen B, Skov M, Garred P, Koch C, Høiby N. Early rise of anti-Pseudomonas antibodies and a mucoid phenotype of Pseudomonas aeruginosa are risk factors for development of chronic lung infection-A case control study. J Cyst Fibros. 2006;5(1):9-15.

34. Levy H, Kalish LA, Cannon CL, Garcia KC, Gerard C, Goldmann D, et al. Predictors of Mucoid Pseudomonas Colonization in Cystic Fibrosis Patients. Pediatr Pulmonol. 2008:43(5):463-71.

\section{Submit your next manuscript to BioMed Central and we will help you at every step:}

- We accept pre-submission inquiries

- Our selector tool helps you to find the most relevant journal

- We provide round the clock customer support

- Convenient online submission

- Thorough peer review

- Inclusion in PubMed and all major indexing services

- Maximum visibility for your research

Submit your manuscript at www.biomedcentral.com/submit
) Biomed Central 